\title{
Rice Stress-Resistant SNP Database
}

Samuel Tareke Woldegiorgis ${ }^{1+}$, Shaobo Wang ${ }^{1+}$, Yiruo He ${ }^{2}$, Zhenhua $\mathrm{Xu}^{1}$, Lijuan Chen ${ }^{1}$, Huan Tao ${ }^{1}$, Yu Zhang ${ }^{1}$, Yang Zou ${ }^{1}$, Andrew Harrison ${ }^{3}$, Lina Zhang ${ }^{1}$, Yufang $\mathrm{Ai}^{1}$, Wei Liu ${ }^{1 *}$ and Huaqin $\mathrm{He}^{1 *}$ (D)

\begin{abstract}
Background: Rice (Oryza sativa L.) yield is limited inherently by environmental stresses, including biotic and abiotic stresses. Thus, it is of great importance to perform in-depth explorations on the genes that are closely associated with the stress-resistant traits in rice. The existing rice SNP databases have made considerable contributions to rice genomic variation information but none of them have a particular focus on integrating stress-resistant variation and related phenotype data into one web resource.

Results: Rice Stress-Resistant SNP database (http://bioinformatics.fafu.edu.cn/RSRS) mainly focuses on SNPs specific to biotic and abiotic stress-resistant ability in rice, and presents them in a unified web resource platform. The Rice Stress-Resistant SNP (RSRS) database contains over 9.5 million stress-resistant SNPs and 797 stress-resistant candidate genes in rice, which were detected from more than 400 stress-resistant rice varieties. We incorporated the SNPs function, genome annotation and phenotype information into this database. Besides, the database has a userfriendly web interface for users to query, browse and visualize a specific SNP efficiently. RSRS database allows users to query the SNP information and their relevant annotations for individual variety or more varieties. The search results can be visualized graphically in a genome browser or displayed in formatted tables. Users can also align SNPs between two or more rice accessions.
\end{abstract}

Conclusion: RSRS database shows great utility for scientists to further characterize the function of variants related to environmental stress-resistant ability in rice.

Keywords: SNP, Biotic stress, Abiotic stress, Database

\section{Background}

Rice (Oryza sativa L.) is one of the significant cereal crops, feeding a large number of worldwide populations. It is also one of the well-studied model organisms for plant research. The need to breed robust and high-productivity rice varieties is more critical than ever due to increasingly adverse environmental conditions and scarce natural resources. Rice yield is limited inherently by environmental stresses, including biotic and abiotic stresses. Rice breeders try to limit yield losses from environmental stresses by incorporating resistant genes and developing more climatically resilient cultivars. Thus, it is of great importance to perform in-depth explorations on the genes that are closely associated with the stress-resistant traits in rice.

\footnotetext{
* Correspondence: weilau@fafu.edu.cn; hehq3@fafu.edu.cn

'Samuel Tareke Woldegiorgis and Shaobo Wang contributed equally to this work.

${ }^{1}$ College of Life Sciences, Fujian Agriculture and Forestry University, Fuzhou 350002, China

Full list of author information is available at the end of the article
}

The recent advances in rice genome biology has generated a tremendous amount of valuable data, including a high-quality reference genome provided by the MSU Rice Genome Annotation Project (Ouyang et al. 2007) and the International Rice Genome Sequencing Project's (IRGSP) RAPdb (Kawahara et al. 2013; Sakai et al. 2013) and genome re-sequencing data of 3010 rice accessions in the rice germplasm core collection ( $\mathrm{Li}$ et al. 2014). The achievement of those rice genome datasets and the discovery of large numbers of single nucleotide polymorphisms (SNPs) in genome-scale sequencing initiatives opening new doors into the study of the genome-wide distribution of diversity, the design of molecular markers for genetic mapping of quantitative trait loci (QTLs) or genes, and the evolutionary dynamics of rice genomes (Feltus et al. 2004).

The optimal management of the huge amount of biological information requires the implementation of dedicated bioinformatics facilities. For this reason, a number of integrative databases and web resources for rice genomic 
variations had been created and curated. IC4R Rice Variation Database collected almost all the available genome resequenced data for different rice varieties, identified about $18 \mathrm{M} \mathrm{SNPs}$ and curated the SNP effects by integrating available genotype-to-phenotype association results (Zhang et al. 2016). RiceVarMap is another comprehensive database for rice genomic variation and its functional annotation (Zhao et al. 2015). Rice SNP-Seek Database (Alexandrov et al. 2015; Mansueto et al. 2017), which resulted from 3000 Rice Genomes Project, provided genotype, phenotype and variety information for rice. SNP haplotype database is a haplotype map database which curated validated SNP information collections from around the world with a specific focus on Japanese rice collections (Yonemaru et al. 2014). Most of these databases focused mainly on overall variation from a vast set of rice varieties. On the other hand, some databases have been designed with particular focus on specialized rice genomic data, such as OryzaGenome (Ohyanagi et al. 2016), a genome variation database for 21 wild Oryza species and 446 O. rufipogon varieties. STIFDB2 (Stress Responsive Transcription Factor Database), is a database for transcription factors and consensus binding sites of stress-responsive genes in Arabidopsis and rice (Naika et al. 2013), while PRGdb is a bioinformatics platform for plant resistant gene analysis (Sanseverino et al. 2010; Osuna-Cruz et al. 2018).

Although the existing related databases have made considerable contributions to rice genomic variation information, none of them presented the rice genomic variations based on special traits such as rice stress resistance/susceptibility. Additionally, the existing SNP databases have not yet incorporated the variants for some of the rice stress resistant varieties. On the other hand, the existing rice stress phenotype databases provide only the phenotypic data of the stress resistant/varieties. Therefore, the we reason the importance of developing a dedicated database for rice stress resistant SNPs, which accommodates the phenotypic data and genomic variations of rice stress resistant varieties in one unified bioinformatics platform would greatly benefit rice researchers. The availability of such an interactive database to explore genomic variability in rice stress-resistant varieties will facilitate research studies, provide scientists a handy tool to access information regarding genetic variants of abiotic and biotic stress resistant rice varieties and help them to identify potential candidate genes for rice stress-resistance.

In this paper, we present a database, the RSRS database (http://bioinformatics.fafu.edu.cn/ RSRS), which is a specifically designed database to present the genomic variation of abiotic and biotic stresses-resistant rice varieties. We first collected the genomic re-sequencing data of over 400 rice varieties with different resistant ability to different biotic and abiotic stresses from previous researches, detected the SNPs in these rice variety genomes, and then screened the stress-resistant SNPs in the resistant rice varieties and catalogued these SNPs in a database system.

\section{Materials and Methods}

\section{Data Collection and Organization}

To compile the rice stress-resistant SNPs dataset, we first collected the stress resistant/susceptible rice varieties and their genomic re-sequencing data from previously

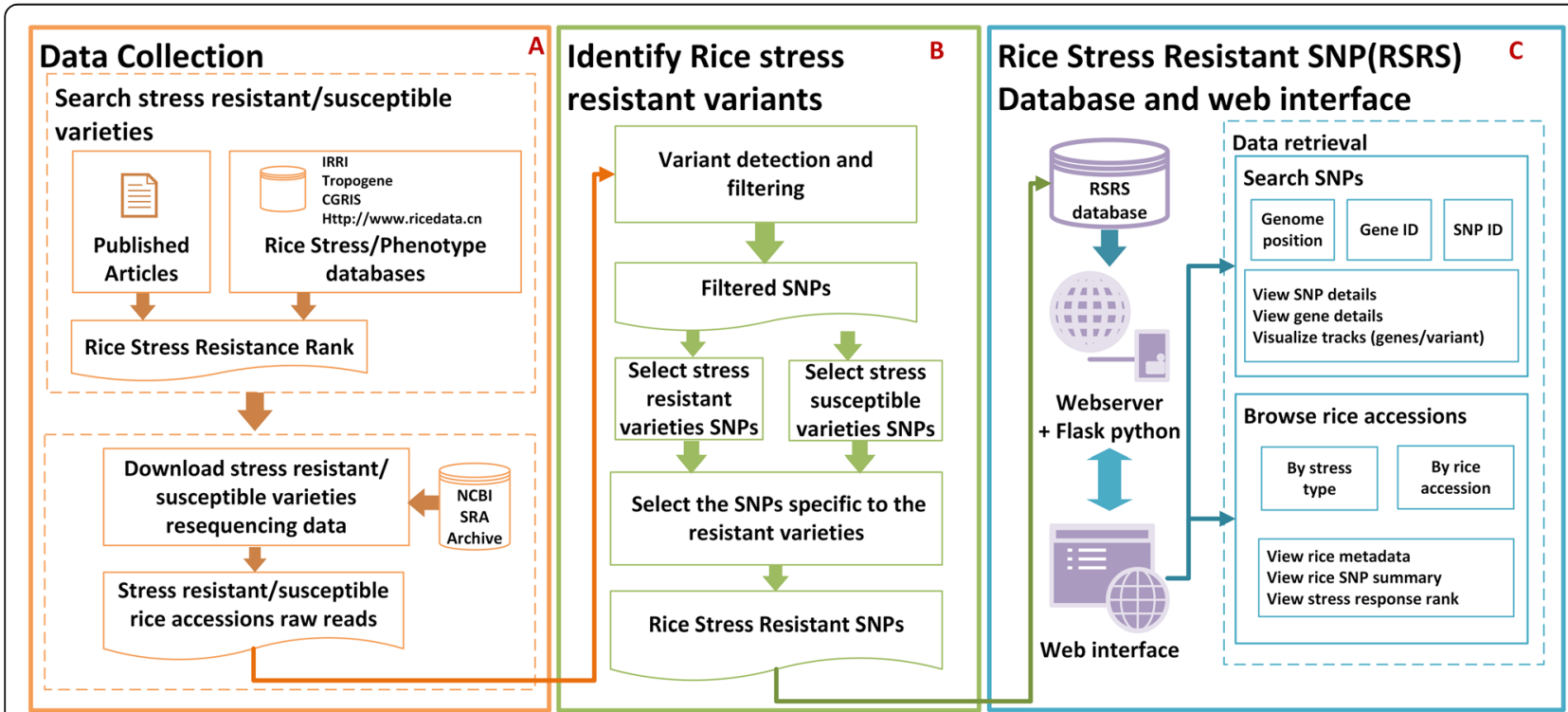

Fig. 1 System view of RSRS database (a) Data collection of rice stress resistant/susceptible varieties and their re-sequencing data (b) Variant detection of the rice stress resistant/susceptible varieties and identification of the stress resistant specific SNPs (c) The RSRS database and web interface design layout 
conducted studies and publications (Fig. 1a). In this study, we collected the biotic stress- (rice blast fungus, bacteria, and pest) or abiotic stress- (heat, cold, flood, salt, alkali and zinc) resistance rank values for different varieties. The stress-responsiveness of each variety is ranked as Highly Resistant (HR), Resistant (R), Moderately Resistant (MR), HS (Highly Susceptible), Moderately Susceptible (MS) and Susceptible (S).

To provide a coherent view for the users, the RSRS database was organized on the basis of different stress types. Under each stress type, the list of stress-resistant rice varieties, metadata and their resistance rank was provided. The corresponding SNPs were also categorized under each stress group so that each SNP site in different rice varieties could be presented with its allelic frequency, annotation, the associated gene and GWAS supporting data if available.

\section{SNP Calling and Annotation}

The variant calling pipeline followed the best practices of SNPs and Insertion/deletion (indel) discovery using Genomic Analysis Toolkit (GATK) v3.8 (Van der Auwera et al. 2013). This pipeline accepts genomic re-sequencing pairedend reads in SRA or FASTQ formats. The processing workflow was created using the snakemake workflow system (Köster and Rahmann 2012).

The quality check for the re-sequencing data of all the tested rice varieties was first conducted and the lowquality reads were trimmed using the Fastp trimming tool (Chen et al. 2018). After trimming, the clean reads were aligned to the rice reference genome (Nipponbare, IRGSP 1.0) (Kawahara et al. 2013) with Burrows-Wheeler Aligner (BWA) (Li and Durbin 2009) and indexed with SAMtools (Li et al. 2009). The aligned reads were sorted using Picard SortSam tool. Samples of each variety were merged using the SAMtools merge tool. The merged reads were deduplicated and read groups were added using the Picard MarkDuplicates and AddOrReplaceReadGroups tools, respectively (Broad Institute 2019). Subsequently, the GATK tools were used to recalibrate the base quality scores to obtain more accurate quality scores for each base.

Variant calling was then performed with a minimum Phred-scaled confidence threshold of 30 using the GATK-HaplotypeCaller (Van der Auwera et al. 2013) in GVCF mode to emit a GVCF file of each variant, and GATK-GenotypeGVCFs tool genotyped the variant files with the multi-sample model. Finally, the raw variants generated from the variant calling steps were further filtered to identify the high quality variants by using the GATK-VariantFiltration tool with the GATK recommended filtering criteria, QD $<2.0$, FS $>60.0, \mathrm{MQ}<40.0$, MQRankSum $<-12.5$ and ReadPosRankSum $<-8.0$, SOR > 4.0 (Van der Auwera et al. 2013).

\section{Identification of Stress-Resistant SNPs in Rice}

After detecting the SNPs of all the tested varieties, we screened the stress-resistant SNPs based on the approach introduced by Silva et al. (2012) and Li et al. (2017). First, we detected the SNPs from the resistant and susceptible rice varieties using the variant detection pipeline mentioned in the SNP calling and annotation section. Second, we filtered out all the variants with read depth less than 3. Third, we grouped the SNPs into two categories under each stress type, one was detected from the stress-resistant rice varieties and the other was from the susceptible rice varieties. Fourth, we selected all the variants, which were detected in three or more stress-susceptible rice varieties. Fifth, we filtered out all the common variants observed between the resistant and susceptible groups and selected the SNPs unique to the resistant varieties (Fig. 1b). Finally, the effect of each variant on its target gene was predicted and annotated using the SnpEff 4.3r program (Cingolani et al. 2012), the SNPs were then classified based on their genomic region and variant effect.

In this study, we mainly focused on non-synonymous SNPs (nsSNPs) and other major effect SNPs as they could lead to amino acid residue change and alter the functional or structural properties of the target protein. Furthermore, we classified the genes associated with non-synonymous variants based on their functional annotation using the agrigo online functional annotation toolkit (Tian et al. 2017). The Physical positions and annotations of the rice genes were retrieved from RAP (https://rapdb.dna.affrc.go.jp/) databases. The GO terms and assignments for rice genes were downloaded from Gramene database (http://www.gramene.org/). The RAP rice gene locus IDs in our database were converted to MSU IDs using the RAP-DB ID converter tool (http:// rapdb.dna.affrc.go.jp/tools/converter).

\section{Database Design and Web-Application Architecture}

Three software components, including an Apache webserver (https://httpd.apache.org/), MySQL 5.7 relational database (http://www.mysql.com/) and Flask web development library (http://flask.pocoo.org/), were used to construct the RSRS database server-side (Fig. 1c).

In the RSRS database, nine MySQL tables were set up, including a 'SNP_genotype' table, a 'SNP_annotation' table, a 'Gene_info' table, a 'GO_annotation' table, a 'GWAS_Info' table, three phenotype data tables and a 'Pheno_SNP' table. The phenotype data tables stored the rice variety and stress related information, while the 'Pheno_SNP' table stored SNPs associated with each stress type. 'var_ID' uniquely identified each variety and 'pheno_ID' was defined to group the varieties resistant/ susceptible to each stress type category. These two keys were used to link the phenotype data tables. The 'SNP_ genotype' table stored the chrom, position, reference and 
genotypes of each SNP retrieved from the variant detection result files. Each SNP may have more than one allele. The annotation of each SNP allele is stored in the 'SNP_annotation' table. 'SNPID' was defined to identify each SNP uniquely and it is the key used to connect the SNP_genotype table with the SNP_annotation table. For efficient storage and data retrieval, the genotypes of each SNP were stored as JSON datatype. The 'Gene Info' and 'GO_annotation' tables were used to store the detail of each gene and gene ontology annotations downloaded from the rice annotation database (https:// rapdb.dna.affrc.go.jp/) and gramene database (http:// www.gramene.org) respectively. The 'gene_ID' was defined as a primary key in 'Gene_Info' table, it linked this table to the SNP_annotation table to relate the associated genes with each SNP. The 'GWAS_info' table stored the GWAS records associated with the SNPs in each stress type. The entity-relationship (ER) diagram of the RSRS database is shown in Fig. 2.

RSRS web service was implemented in Python (https:// www.python.org/) and based on the Flask web development library (http://flask.pocoo.org/). The Web Services consist of the phenotype and genotype data retrieval and provides the visualization tools to display the search results. The searchable terms include phenotype name, SNP ID, gene ID and chromosome region (Fig. 1c). The user's query was passed from the front-end, the server initiated and displayed the output from the database module script. All the extracted information was displayed in a tabular format.

The interactive user interface was developed using the Bootstrap3 (http://www.getbootstrap.com) framework which combines HTML, CSS and JavaScript. Visualization of the

\begin{tabular}{|c|c|c|c|}
\hline Variety_Info & SNP_annotation & \multicolumn{2}{|l|}{ Gene_Info } \\
\hline PK $\quad$ var_ID & \multirow{2}{*}{$\begin{array}{l}\text { snp_ID } \\
\text { allele }\end{array}$} & \begin{tabular}{l|l} 
PK & gene_ID
\end{tabular} & \\
\hline accession_ID & & \multicolumn{2}{|l|}{ gene_symbol } \\
\hline var_name & annotations $(\ldots .)$. & \multicolumn{2}{|l|}{ chrom } \\
\hline \multirow{2}{*}{$\begin{array}{l}\text { var_population } \\
\text { var_country }\end{array}$} & gene_ID & \multicolumn{2}{|l|}{ start_pos } \\
\hline & & \\
\hline & & \\
\hline & SNP_genotypes & & \\
\hline Phenotype_Data & \begin{tabular}{l|l} 
PK & snp_ID \\
\end{tabular} & \multirow{2}{*}{\multicolumn{2}{|c|}{ GO annotation }} \\
\hline var_ID & chrom & & \\
\hline \multirow{2}{*}{ pheno_ID } & & \begin{tabular}{l|l} 
PK & go_ID \\
\end{tabular} & \\
\hline & \multirow{2}{*}{$\begin{array}{l}\text { REF } \\
\text { genotypes(json) }\end{array}$} & go_domain & \\
\hline $\begin{array}{l}\text { stress_Rank } \\
\text { study_reference }\end{array}$ & & go_name & \\
\hline & Pheno_SNP & GWAS_info & \\
\hline Phenotypes & \multirow{2}{*}{$\begin{array}{l}\text { snp_ID } \\
\text { pheno_ID }\end{array}$} & snp_ID & \\
\hline \begin{tabular}{l|l} 
PK & pheno_ID \\
\end{tabular} & & pheno_ID & \\
\hline \multirow{2}{*}{$\begin{array}{l}\text { pheno_name } \\
\text { type }\end{array}$} & \multirow[t]{2}{*}{ minor_allele_frequency } & \multirow{2}{*}{$\begin{array}{l}\text { p_val } \\
\text { study_reference }\end{array}$} & \\
\hline & & & \\
\hline \multicolumn{4}{|c|}{$\begin{array}{l}\text { Fig. } 2 \text { Entity-Relationship Diagram for the RSRS database. The Pheno_SNP table is the table, which connects the phenotype details with the genotype } \\
\text { details. In this table the pheno_ID field is used to identify the associated stress with the varieties and the snp_ID is used to identify the associated SNPs } \\
\text { with the varieties. The Pheno_SNP table have a 1:N relationship with the Phenotypes and SNP_genotypes tables using pheno_ID and snp_ID keys. The } \\
\text { SNP_genotypes table describes the detail of each SNP, which is characterized by its own unique ID, snp_ID, chrom, position, reference allele and } \\
\text { genotypes of each accession. A SNP may consist of multiple alleles in which the annotation of each allele is stored in the SNP annotation table. The } \\
\text { SNP_annotation table is connected in a 1:N relationship with the Gene_info table, which stores the detail of each gene. The Gene_Info table is } \\
\text { connected to the GO_annotation table to characterize the associated genes with each SNP. The variety and phenotype details are stored in } \\
\text { Variety_Info, Phenotype_data and Phenotypes table, var_ID and pheno_ID are used to connect these tables. The GWAS_info table stores the } \\
\text { associated GWAS record of each SNP with its associated stress. The ER diagram was created by using draw.io at https://Www.draw.io/ }\end{array}$} \\
\hline
\end{tabular}


data via statistical graphs and the tabular view was implemented using JavaScript charting libraries called Highcharts (https://www.highcharts.com/) and Datatables (https://data tables.net/), respectively. We hosted the system on a server running Ubuntu 16.04LTS powered by Apache server (Apache Version 2.4).

\section{Results and Discussion \\ Database Content}

The whole genome sequencing data of 402 rice (Oryza sativa L.) varieties with different abiotic and biotic stress-resistant ability were downloaded from the 3000 rice genome re-sequencing project and other projects in NCBI SRA database. Following the protocol introduced in Fig. 1, the abiotic and biotic stress-resistant rice varieties and their SNPs were detected and stored in the RSRS database.

\section{Phenotype Data}

The information for each variety including country of origin, ID, variety name and variety group was collected from the IRIC information site (http://iric.irri.org/resources/3 000-genomes-project) and other related studies. The accession ID and the ID used for the variety is shown in the Additional file 1: Table S1. Additionally, we also collected the stress responsiveness of all the tested varieties from previous studies and phenotype databases. The list of the varieties in each stress group and the detailed information of each variety including the related references is also shown in the Additional file 1: Table S1.
SNP Data After detecting the genetic variants in each tested rice variety genome and filtering the stress-resistant SNPs from the stress-resistant rice varieties, more than 9.5 million stress-resistant SNPs in rice were identified and stored in the RSRS database. Table 1 summarizes the number and effect of the stress-resistant SNPs located in the different genomic regions, including genic, intergenic, intronic and upstream/downstream regions. The stressresistant SNPs located in particular positions, such as start and stop codons, splice donor and acceptor sites, are also displayed in Fig. 3.

\section{Biotic Stress-Resistant SNPs}

In the biotic stress-resistant SNPs category, there are three sub-group SNPs, which are resistant to 3 kinds of biotic stresses, including fungus, bacteria and pest infection.

\section{Blast Fungus-Resistant SNPs}

A final set of 6,785,349 SNPs were called from 152 blastresistant rice varieties using Nipponbare as the reference genome. We detected 3,324,526 SNPs from 26 highly blast-susceptible rice varieties using the same pipeline. After screening the SNPs specific to the blast-resistant varieties, 3,638,230 potential blast-resistant SNPs were detected and stored in this sub-group.

In these blast-resistant rice varieties, we identified 24,352 genes harboring blast-resistant nsSNPs, which were regarded as potential blast-resistant genes in rice. Based on the functional annotation of these genes, 609 of the potential blastresistant genes were associated with stress stimuli and

Table 1 Number of stress-resistant SNPs located in different regions of rice genome

\begin{tabular}{|c|c|c|c|c|c|c|c|}
\hline Stress types & $3^{\prime} U T R^{b}$ & $5^{\prime} \mathrm{UTR}^{\mathrm{b}}$ & Downstream $^{\text {b }}$ & Intergenic $^{b}$ & Intron $^{b}$ & $\mathrm{CDS}^{\mathrm{a}}$ & Upstream $^{\mathrm{b}}$ \\
\hline Heat Stress & 93,767 & 55,759 & 653,058 & 635,265 & 63,162 & 205,078 & $2,780,114$ \\
\hline Alkali Stress & 66,920 & 37,644 & 388,645 & 331,550 & 43,425 & 147,100 & $1,755,014$ \\
\hline Salt Stress & 79,546 & 44,882 & 470,148 & 409,469 & 51,300 & 175,835 & $2,081,462$ \\
\hline Flood Stress & 67,419 & 37,926 & 391,338 & 334,562 & 43,764 & 142,223 & $1,787,105$ \\
\hline Cold Stress & 58,798 & 33,903 & 370,763 & 332,310 & 40,007 & 126,918 & $1,612,926$ \\
\hline Zinc Stress & 74,843 & 41,853 & 411,990 & 330,483 & 48,027 & 161,716 & $1,908,842$ \\
\hline Blast Fungus & 81,491 & 47,765 & 522,328 & 480,785 & 53,365 & 183,547 & $2,256,982$ \\
\hline Bacteria Leaf Blight & 63,649 & 35,647 & 368,539 & 319,163 & 41,456 & 138,399 & $1,633,392$ \\
\hline Bacteria Sheath Blight & 51,663 & 29,237 & 331,287 & 272,583 & 34,716 & 112,617 & $1,356,611$ \\
\hline Bacterial Rice Planthopper & 72,686 & 41,112 & 417,411 & 347,624 & 45,912 & 155,346 & $1,890,893$ \\
\hline Bacteria Stripe leaf blight & 49,221 & 27,107 & 269,081 & 203,861 & 31,815 & 103,938 & $1,274,279$ \\
\hline Brown Planthopper Pest & 79,293 & 44,994 & 475,951 & 410,522 & 52,299 & 173,293 & $2,109,723$ \\
\hline Gall Midge Pest & 50,495 & 29,177 & 291,121 & 230,150 & 32,704 & 107,667 & $1,367,183$ \\
\hline Small Brown Planthoppers Pest & 86,755 & 47,381 & 465,586 & 363,512 & 55,723 & 182,484 & $2,205,066$ \\
\hline Whitebacked Planthopper Pest & 94,541 & 53,730 & 552,635 & 462,375 & 61,037 & 203,404 & $2,508,144$ \\
\hline Rice Leafroller Pest & 79,704 & 44,658 & 448,760 & 358,452 & 50,581 & 172,603 & $2,072,717$ \\
\hline
\end{tabular}

${ }^{a}$ CDS includes the SNPs in exonic region including nsSNPs (non-synonymous SNPs) and sSNPs (synonymous SNPs)

' Intergenic, 5'UTR, Intron 3'UTR, Upstream and Downstream indicate that No. of stress-resistant SNPs located in the Intergenic, 5'UTR, Intronic, 3' UTR, upstream and downstream regions of a gene 


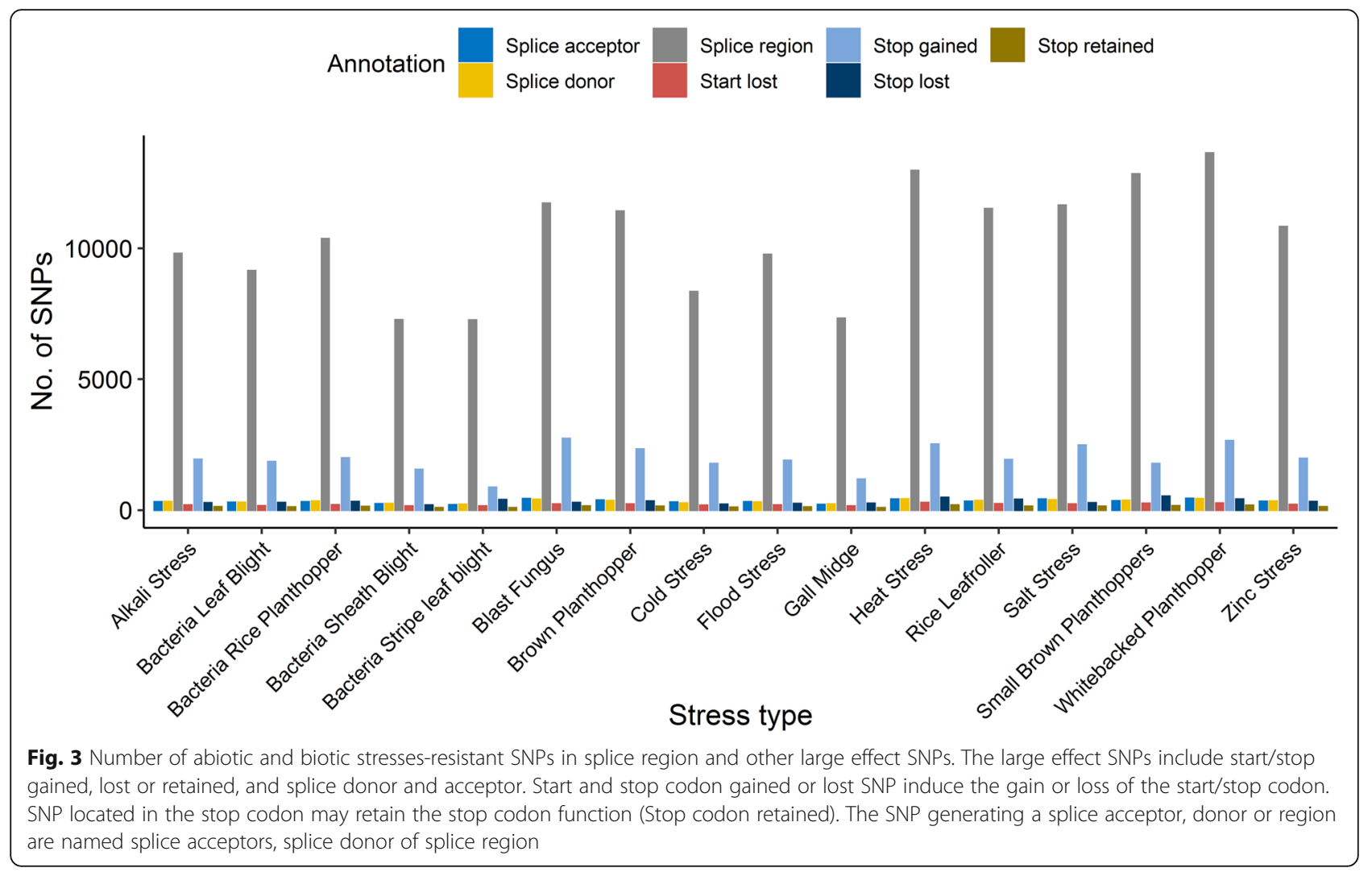

response ability. Interestingly, 139 of them had been reported to function as blast-resistant genes in different references, which were shown in Additional file 2: Table S2. Among the 139 known blast-resistant genes, 24 genes were transcription factors, 34 genes were disease resistance genes, and the other 81 genes were involved in different families of stress-response abilities. In total, there were 8005 stress-resistant SNPs on these 139 genes, in which $11.5 \%$ were missense, $67.0 \%$ were in the upstream/downstream region, $8.6 \%$ were synonymous variants and $12.0 \%$ include the others (Fig. 5). These variants were distributed in the rice varieties with allele frequency between 0.01 and 1.0 (Fig. 4).

\section{Bacterial Stress-Resistant SNPs}

We first collected 74 bacterial-resistant rice varieties, including 41 bacterial leaf blight-resistant varieties with a total of 4,307,743 SNPs, 18 bacteria sheath blightresistant varieties with a total of 3,991,441 SNPs, 5 bacteria stripe leaf blight resistant varieties with a total of 1 , 971,323 SNPs and 24 bacteria planthopper-resistant varieties with a total of 4,242,547 SNPs. Similarly, we had 8 bacterial leaf blight-susceptible varieties with 1,856,574 SNPs, 7 bacterial sheath blight-susceptible varieties with 1,999,162 SNPs, 6 bacterial rice planthopper-susceptible varieties with 1,344,685 SNPs and 3 bacteria stripe leaf blight-susceptible varieties with a total of 523,829 SNPs.
Finally, we generated 2,608,978, 2,176,113, 2,980,714 and $1,965,432$ SNPs resistant to bacterial leaf blight, sheath blight, rice planthopper and stripe leaf blight in the rice genome, respectively.

We identified 19,154,17,711, 20,972 and 17,987 genes harbouring bacterial leaf blight, sheath blight, rice planthopper, and stripe leaf blight-resistant nsSNPs, respectively. Among them 483, 433, 518 and 447 were associated with stress stimuli and responses based on the functional annotation. Interestingly, 64 bacterial leaf blight, 11 bacterial sheath blight, 22 bacterial rice planthopper and 7 rice stripe disease-resistant potential genes had been confirmed in different publications, shown in Additional file 2: Table S2. These genes included 15 transcription factors, 19 disease resistance genes and others associated with defence response activities. The bacterial stress-resistant SNPs distributed on these genes consist of $7.43 \%$ nsSNPs, $6.78 \%$ synonymous SNPs and 79.5\% upstream/downstream (Fig. 5). The allelic frequency distribution of the SNPs in these genes varies between 0.022 and 1.0 (Fig. 4). Additionally, (Zhang et al. 2017) verified 121 significantly enriched SNPs which contributed to bacterial leaf blight-resistance in rice. Among them, 91 were mapped to the bacterial leaf blight-resistant SNPs in the RSRS database. In another research work, 46 SNPs were found to be associated with bacterial blightresistance in rice (Dilla-Ermita et al. 2017), among which 29 were also detected in the RSRS database. 


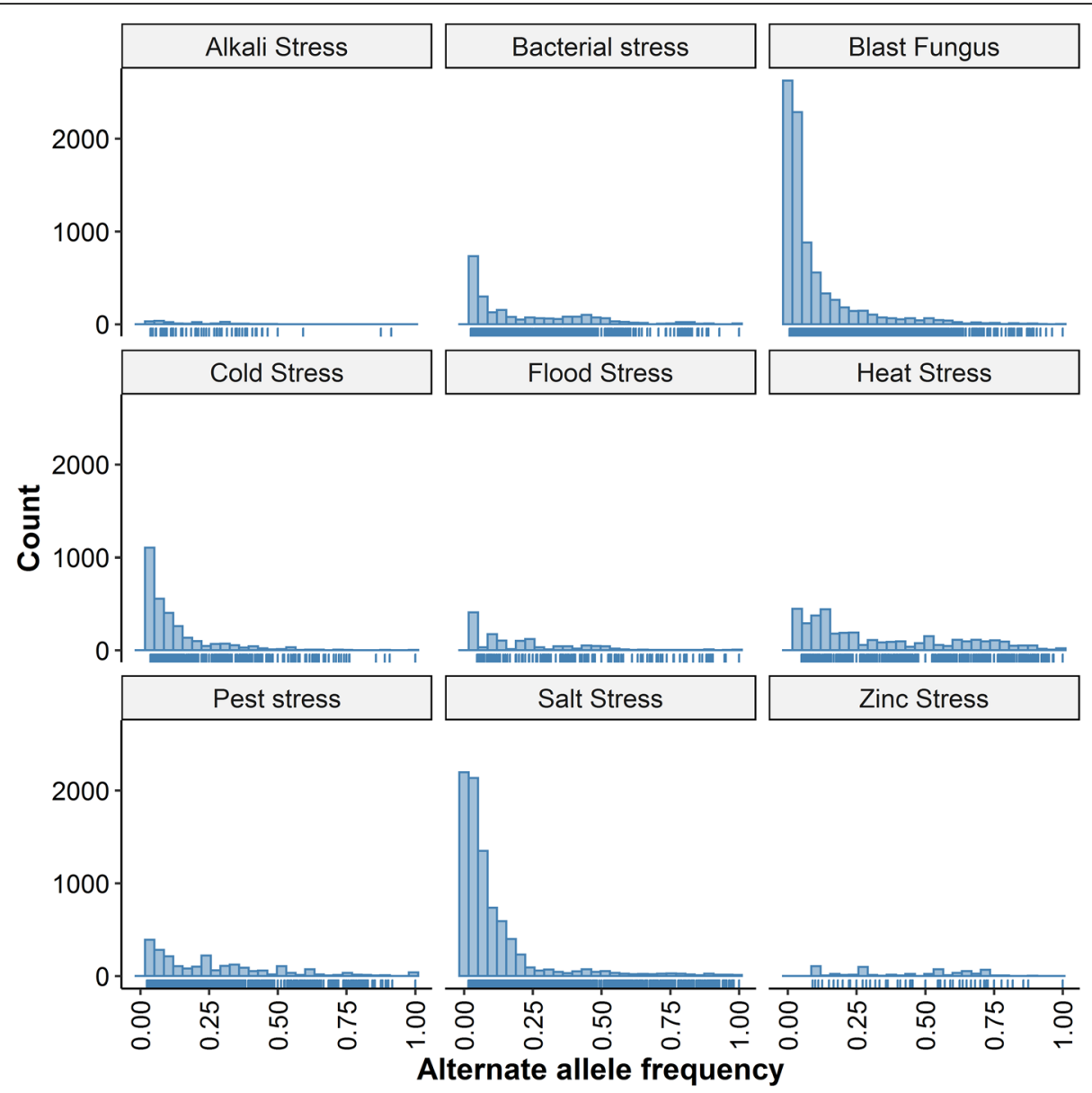

Fig. 4 The variants distribution by annotation in different abiotic and biotic stress-resistant genes in rice. The X-axis represents the variant annotation of the SNPs in these genes and the Y-axis represents the count of each annotation in these genes

\section{Pest Stress-Resistant SNPs}

A total of 81 pest-resistant rice varieties were identified, including 45 brown planthopper pest-resistant varieties with 6,054,118 SNPs, 4 gall midge pest-resistant varieties with 3,638,406 SNPs, 8 small brown planthopper pestresistant varieties with 4,221,428 SNPs, 35 white backed planthopper pest-resistant varieties with $5,957,813$ SNPs and 10 rice leafroller pest-resistant varieties with 4,679 , 556 SNPs. Similarly, we detected 3,710,640 SNPs from 8 brown planthopper-susceptible rice varieties, 4,637,279 SNPs from 3 gall midge susceptible varieties, 2,392,253 SNPs from 3 small brown planthopper-susceptible varieties, 3,362,269 SNPs from 6 white backed planthoppersusceptible varieties, and 3,055,842 SNPs from 3 leaf roller pests-susceptible varieties. Finally, we found 3,357,284 brown planthopper, 2,115,252 gall midge, 3,417,213 small brown-, 3,948,989 white backed planthopper- and 3,237, 416 rice leafroller pest-resistant SNPs in rice, respectively.

In the pest-resistant varieties, we identified a total of 22 , $876,19,782,23,721,24,098,22,101$ genes harboring nsSNPs in brown planthopper pest, gall midge pest, small-brown planthopper pest, white-backed planthopper pest and rice leafroller pest-resistant rice varieties respectively. We classified these genes based on their functional annotation and we found that 555, 489, 614, 603 and 540 genes were associated with stress and stimuli response related functions. Among them, 29 genes have been reported to be involved in pest caused diseases resistance, which is shown in the Additional file 2: Table S2. These genes were constituted of 3 transcription factors, 14 pathogen resistance genes and other defence related functions. The total number of SNPs detected in these genes included $72.1 \%$ of upstream/downstream, $14 \%$ of nsSNPs and 7.08 of synonymous SNPs (Fig. 4). The allelic diversity of the SNPs varies within the range of 0.022 to 1 (Fig. 5).

\section{Abiotic Stress-Resistant SNPs}

In the abiotic stress category, we detected SNPs in the genome of rice varieties with resistant ability to heat, alkali, salt, cold, flood and zinc stresses. 


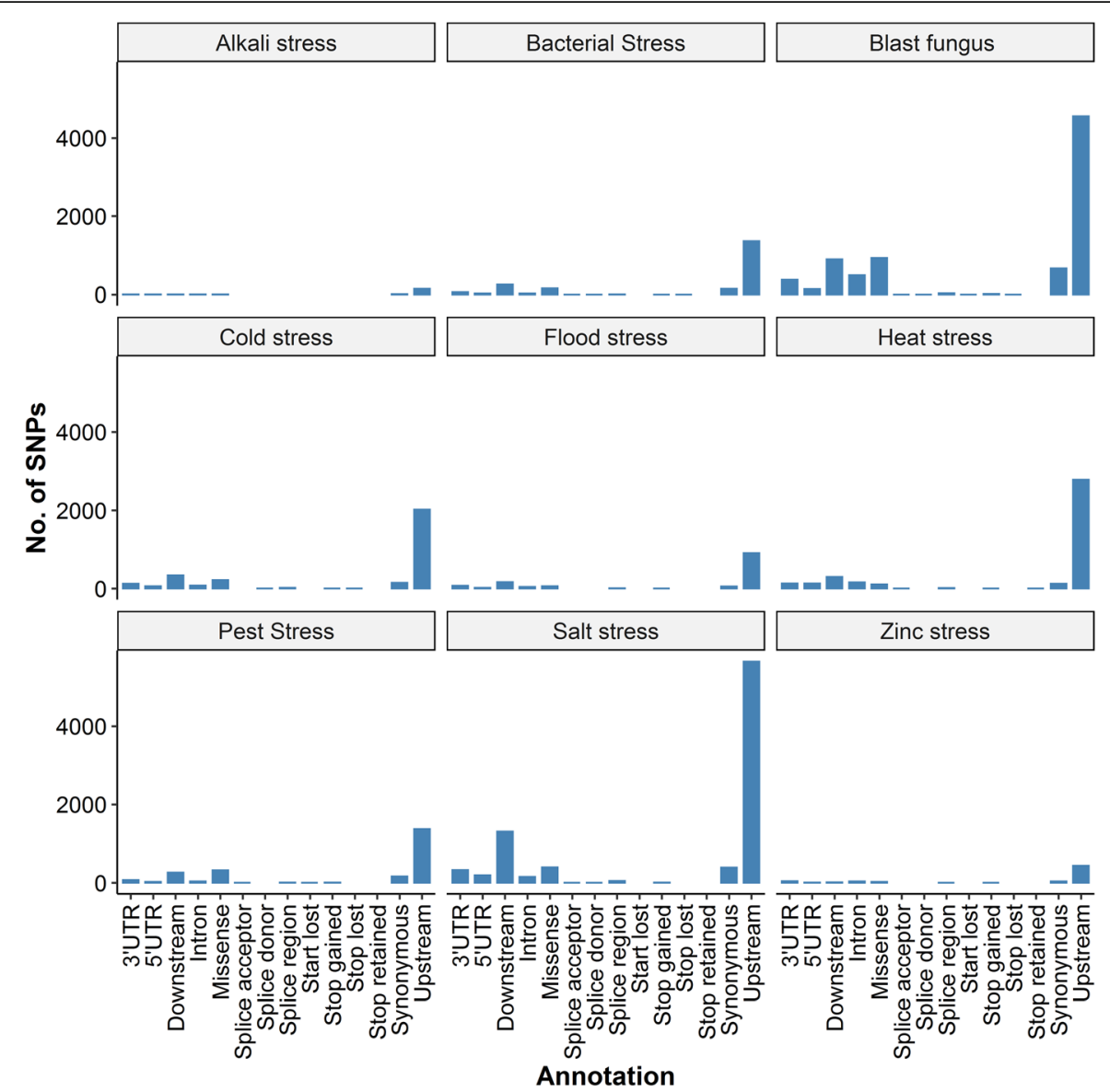

Fig. 5 The alternate allelic frequency distribution in different abiotic and biotic stress-resistant genes in rice. The $X$ axis represents the allele frequencies of the non-reference alleles and the $Y$ axis represents of the count of each allele frequency of the SNPs in these genes

\section{Heat Stress-Resistant SNPs}

Twenty heat stress-resistant rice varieties were used in this study and a total of 5,707,672 SNPs were detected from this set of rice varieties. Similarly, we detected 1, 233,652 SNPs from 8 highly heat-susceptible rice varieties. Finally, a total of 4,500,192 heat stress-resistant specific SNPs in rice were identified.

In the genome of heat stress-resistant rice varieties, we identified a total of 26,357 genes harbouring heat stressresistant nsSNPs. Based on the functional annotation, 661 genes of them were associated with stress response and stimuli. Interestingly, 75 genes had been reported to be involved in heat stress tolerance in rice. Among these 75 genes, 6 genes were transcription factors and 23 are heat shock proteins, which are shown in the Additional file 2: Table S2. The total number of SNPs in these genes was 3745 , including $82 \%$ upstream/downstream, $3 \%$ nsSNPs, $3.2 \%$ synonymous and $12 \%$ other variants. The allele frequency of these variants varied between 0.047 and 1 (Figs. 4 and 5).

\section{Alkali Stress-Resistant SNPs}

Twenty-seven alkali stress-resistant rice varieties were used in this study. The total number of SNPs called from this group was 4,931,032. From the 10 susceptible rice varieties, we detected 2,314,097 SNPs. After filtering out the common variants between the resistant and susceptible varieties, we generated 2,779,427 alkali stress-resistant SNPs in rice.

In this subcategory, we identified a total of 22,876 genes harbouring alkali stress-resistant nsSNPs. Based on the functional annotation, 513 genes were associated with stress response and stimuli. Among them, five genes have been reported to be associated with alkali stress tolerance in rice, which is shown in the Additional file 2: Table S2. The total number of SNPs associated with these genes was 182 and the allele frequency varies between 0.022 and 0.9 (Figs. 4 and 5).

\section{Salt Stress-Resistant SNPs}

Sixty-one salt stress-resistant rice varieties were used in this study. The number of SNPs identified from this set of 
rice varieties was $6,080,805$. Similarly, we detected 2,924, 352 from 17 salt stress-susceptible rice varieties. After filtering out the SNPs in salt stress-susceptible rice varieties, we identified a total of 3,323,770 salt stress-resistant specific SNPs in the salt stress-resistant varieties.

In these salt-stress resistant varieties, we mapped a total of 23,461 genes harbouring salt stress-resistant nsSNPs. Based on the functional annotation, 589 genes were associated with stress response and stimuli. Interestingly, 209 genes have been reported to be associated with salttolerance in rice, including 30 transcription factors, 26 kinase family genes, and 14 ion transporter family, which are also shown in Additional file 2: Table S2. There were a total of 8472 SNPs in these genes, including $4.65 \%$ of missense, $81.4 \%$ of upstream and downstream, $4.6 \%$ of synonymous variants and others (Fig. 5). The allele frequency of these variants varied between 0.012 and 1 (Fig. 4).

In a study conducted by Lekklar et al. (2019), they found 448 significantly enriched SNPs contributing to salt stress-resistant ability in rice by using GenomeWide Association Study (GWAS) method, among which 389 were also detected in the results of this study and 162 were stored as salt stress-resistant SNPs in the RSRS database. Additionally, Jain et al. (2014) screened 910 SNPs present in the coding region of 346 differentially expressed genes from 3 salt stress-resistant rice varieties, of which 862 were consistent with the results of this study and 301 were stored as salt stress-resistant SNPs in the RSRS database.

\section{Cold Stress-Resistant SNPs}

Twenty-seven cold stress-resistant rice varieties were collected in this study, in which we detected a total number of 4,906,148 SNPs. From 10 cold-susceptible rice varieties, we detected a total of 2,560,928 SNPs. Finally, we filtered a total of 2,584,211 cold stress-resistant SNPs in rice.

We mapped 16,217 genes with cold stress-resistant nsSNPs in rice. Based on the functional annotation, 476 genes were associated with stress response and stimuli. Interestingly, 107 genes have been reported to be associated with cold tolerance in rice, which is shown in Additional file 2: Table S2. There were a total of 2998 SNPs located in these genes, in which $88 \%$ were upstream/downstream, $7.2 \%$ were nsSNPs and $4.84 \%$ were synonymous variants (Fig. 5). Their allelic frequency variation ranged from 0.038 to 1.0 (Fig. 4). Furthermore, the data in other publications also supported our results. Wang et al. (2016) found 181 significantly enriched cold stress-related SNPs in rice of which 169 were consistent with the SNPs in the RSRS database (Wang et al. 2016).

\section{Flood Stress-Resistant SNPs}

Twenty-one flood stress-resistant rice varieties with a total of 4,851,632 SNPs and 10 flood stress-susceptible rice varieties with 2,238,878 SNPs were collected in this study. Finally, we identified 2,813,598 flood stress-resistant SNPs in flood stress-resistant rice varieties.

In these flood stress-resistant varieties, we identified a total of 20,818 genes with flood stress-resistant nsSNPs. Based on the functional annotation, 505 genes were associated with stress response and stimuli. Twenty-six of the genes, so far, have been reported to be associated with submergence tolerance, including five transcription factors and five ethylene receptor like proteins, which are also shown in Additional file 2: Table S2. In these 26 genes, we detected 1321 SNPs, of which $80.0 \%$ were upstream/downstream SNPs, $4.47 \%$ were nsSNPs and $4.1 \%$ were synonymous SNPs.

\section{Zinc Stress-Resistant SNPs}

A total of 4,215,431 SNPs were detected in 11 zinc stress-resistant rice varieties, while 1,345,257 SNPs were found in the five zinc stress-susceptible rice varieties. In total 2,987,375 zinc stress-resistant SNPs in rice were identified from the zinc stress-resistant rice varieties.

In these zinc stress-resistant varieties, we identified 20, 818 genes with zinc stress-resistant nsSNPs. Based on the functional analysis, 530 of the genes were associated with stress response and stimuli. Six genes, so far, have been reported to be involved in zinc stress tolerance, which are mainly involved in $\mathrm{Zn}$ uptake and translocation as shown in the Additional file 2: Table S2. In these six genes, there were 538 SNPs, including 74\% upstream/downstream, $4 \%$ nsSNPs, and $6 \%$ Synonymous SNPs (Fig. 5). The allele frequency of these SNPs varied between 0.047 and 0.89 (Fig. 4).

\section{Web Interface}

RSRS is an integrated rice stress resistant SNP database allowing users to explore overall data organization, to obtain information including rice stress related information, stress resistant SNPs and associated genes by querying a specific genomic region, gene ID or SNP ID, and to visualize the data of interest in different output format. The functions of RSRS database were shown in Fig. 1c.

RSRS database allows users to search, compare and browse the phenotypic and genotypic data of the stressresistant rice varieties used in our study. Each rice variety in the RSRS database is assigned with a cultivar ID. The variety browse function presents the phenotype detail of each rice cultivar including the geographical information, population, and the stress-resistance rank. The SNP browse function provides users to bring up the overall SNP summary in each stress-resistant group, SNP summary per chromosome in each cultivar and the distribution of different type of SNPs in each group.

The searching function is a user-friendly web interface for users to query SNP related information. The users 
are able to search for different SNPs by a given SNP ID, gene ID, phenotype, variant annotation, chromosome or a specific genomic region (Fig. 6a).

Searching by genomic region allows users to search for SNPs at a selected chromosome in a given range of genome position. Besides this, retrieval of SNPs using different search options like variant annotation, minor allele frequency can be facilitated in the database.

Searching by SNP ID allows users to search for a specific SNP by using its ID. Each SNP has a unique identifier with a format of SNPCHXXXXXXXX. The first letter of the ID indicates the variant type, such as 'SNP' standing for SNP. The ' $\mathrm{CH}$ ' stands for the chromosome number (01-12) and XXXXXXXX represents chromosome coordinate of a variation.

Searching by gene ID allows the user to search for SNPs associated with a given gene. Similarly, the user can also set the options to search for the variants of a selected annotation or range of minor allele frequencies (Fig. 6a).

The search function results (Fig. 6b) can also be further expanded and the detail of each SNP can be explored. The detail of each SNP includes the chromosome position, the allele distribution, the associated gene information, the variant effects annotation and the genotype of the SNP in each cultivar of the selected phenotype. This function helps the user to figure out the polymorphic SNPs between two or more rice cultivars (Fig. 6c). Additionally, we incorporated the GWAS enrichment scores of the SNPs with published data in this database. The SNPs with their available enrichment score will be displayed in the SNP detail page.

Furthermore, the RSRS database system also integrates the genome browser tool, JBrowse, to navigate the data in a graphical visualization format (Buels et al. 2016). JBrowse allows the users to directly visualize the location of SNPs, display the complete list of annotations, variant details, and genotype data of each accession. The interactive and dynamic genome browser could also display genomic features for each rice variety, gene and the corresponding data tracks, as well as variant alignment (Fig. 6d).

\section{Conclusion}

The initial purpose of the RSRS database provides a convenient way to search and retrieve biotic and abiotic

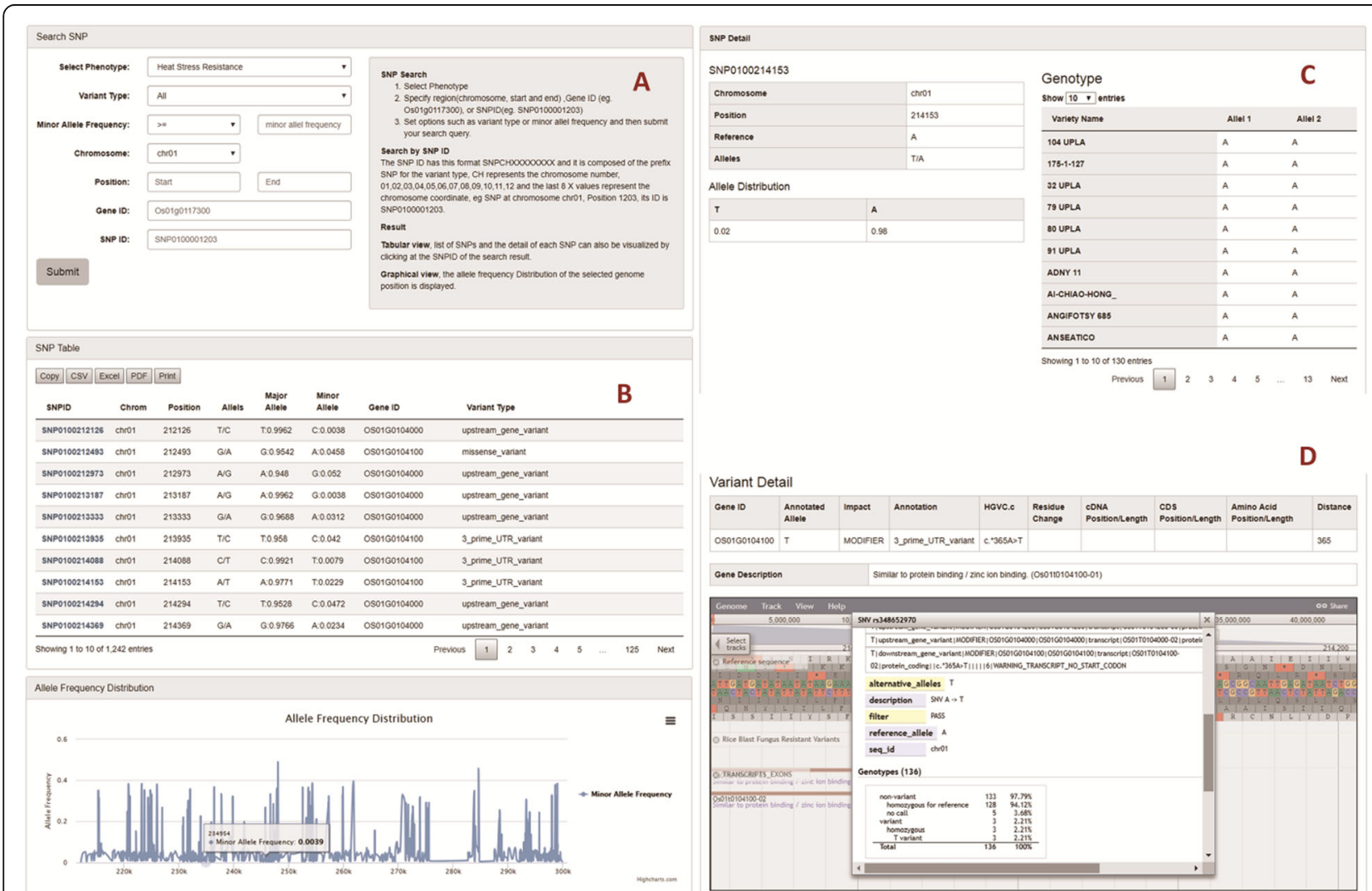

Fig. 6 Examples of search functions in RSRS database. Search Interface (a) allows users to search by region, gene ID and SNP ID. Additionally users can also set options, such as allele frequency, variant types. Search Results (b) retrieves the list of SNPs and displays the allele distribution in a given range. SNP detail view (c) displays the detail of each SNP and the genotype of each rice accession. Jbrowse visualization (d) displays the SNP detail in Jbrowse and gives the detail of the associated gene with the SNPS 
stress-resistant SNPs and their annotation for the rice research community. The RSRS database is built on the genomic re-sequencing data of different rice cultivars with different stresses-resistant ability. In total, over 9.5 million stress-resistant SNPs and 797 genes associated with stress response and stimulus were detected from more than 400 stress-resistant rice varieties. Interestingly, our results were partially supported by previous publications, indicating that the results in this study would give valuable insights for the researchers seeking to identify novel stress-resistant genes in rice.

We organized and presented the stress-resistant SNPs with related data, including genotype and phenotype, in a web-based system. By integrating with comprehensive information, this platform is growing to be a useful tool for a wide range of applications in rice genetics, breeding, and comparative genomics. This database will facilitate the researchers to investigate the genetic variation of the stress-resistant genetic variants in rice in a more streamlined, rapid, and efficient fashion.

We anticipate extending the services of this RSRS database by including more rice accessions with different stress-resistant capability as well as more kinds of stresses. Additionally, we will also make it more comprehensive knowledgebase for rice abiotic and biotic stress-related studies by incorporating other omics data, including transcriptome, proteomics, and metabolomics. We will also endeavour to make the database more user-friendly and more efficient with the suggestion from rice researchers and breeders who make use of this first version of RSRS database.

\section{Supplementary information}

Supplementary information accompanies this paper at https://doi.org/10. 1186/s12284-019-0356-0.

Additional file 1: Table S1. List of Rice Accessions Resistant/Susceptible to Different Biotic Stresses.

Additional file 2: Table S2. List of Genes Involved in Different Biotic/ Abiotic Stress Resistance.

\section{Abbreviations}

GWAS: Genome-Wide Association Study; HR: Highly Resistant; HS: Highly Susceptible; JSON: JavaScript Object Notation; MR: Moderately Resistant; MS: Moderately Susceptible; nsSNP: Non synonymous SNP; QTL: Quantitative Trait Locus; R: Resistant; RSRS: Rice Stress Resistant SNPs; S: Susceptible; SNP: Single Nucleotide Polymorphism; WSGl: Web server gateway interface

\section{Acknowledgments}

The authors thank the anonymous referees whose constructive comments were helpful in improving the quality of this work.

\section{Authors' Contributions}

$\mathrm{HH}$ conceived the study and revised the manuscript. STW and SW carried out the experiment and wrote the manuscript. $Y H, Z X, Y Z$ and $Y Z$ helped to design the RSRS database and website. HT got the $3 \mathrm{~K}$ rice genome resequencing data. LC, LZ and YA helped to mine the biotic and abiotic stresses-resistant genes. $\mathrm{AH}$ and $\mathrm{WL}$ helped to revise the manuscript. The authors have read and approved of the final version.

\section{Funding}

This work was supported by Natural Science Foundation of China and Fujian (No. 31270454; 81502091 and 2016 J01101; 2019 J01060610), Innovative Foundation of FAFU (No. CXZX2017132 and CXZX2017303), Program for Innovative Research Team in College of Life Sciences, FAFU (No. 712018009). The funding body did not participate in the design of the study and collection, analysis, and interpretation of data and in writing the manuscript.

\section{Availability of Data and Materials}

RSRS database is freely available at http://bioinformatics.fafu.edu.cn/RSRS.

Additionally, the data that support the findings of this study are also

provided as supplementary material online.

\section{Ethics Approval and Consent to Participate}

Not applicable.

\section{Consent for Publication}

Not applicable.

\section{Competing Interests}

The authors declare that they have no competing interests.

\section{Author details}

${ }^{1}$ College of Life Sciences, Fujian Agriculture and Forestry University, Fuzhou 350002, China. ${ }^{2}$ School of Science and Engineering, The Chinese University of Hong Kong, Shenzhen 518172, China. ${ }^{3}$ Department of Mathematical Sciences, University of Essex, Colchester, UK.

Received: 24 June 2019 Accepted: 6 December 2019

Published online: 23 December 2019

\section{References}

Alexandrov N, Tai S, Wang W et al (2015) SNP-seek database of SNPs derived from 3000 rice genomes. Nucleic Acids Res 43:D1023-D1027. https://doi.org/ 10.1093/nar/gku1039

Broad Institute (2019) Picard toolkit. Broad Institute, GitHub Repos http:// broadinstitute.github.io/picard/

Buels R, Yao E, Diesh CM et al (2016) JBrowse: a dynamic web platform for genome visualization and analysis. Genome Biol 17:66. https://doi.org/10. 1186/s13059-016-0924-1

Chen S, Zhou Y, Chen Y, Gu J (2018) Fastp: an ultra-fast all-in-one FASTQ preprocessor. Bioinformatics 274100. https://doi.org/10.1101/274100

Cingolani P, Platts A, Wang LL et al (2012) A program for annotating and predicting the effects of single nucleotide polymorphisms, SnpEff. Fly (Austin) 6:80-92. https://doi.org/10.4161/fly.19695

Dilla-Ermita CJ, Tandayu E, Juanillas VM et al (2017) Genome-wide association analysis tracks bacterial leaf blight resistance loci in Rice diverse Germplasm. Rice 10:8. https://doi.org/10.1186/s12284-017-0147-4

Feltus FA, Wan J, Schulze SR et al (2004) An SNP resource for Rice genetics and breeding based on subspecies Indica and japonica genome alignments. Genome Res:1812-1819. https://doi.org/10.1101/gr.2479404

Jain M, Moharana KC, Shankar R et al (2014) Genomewide discovery of DNA polymorphisms in rice cultivars with contrasting drought and salinity stress response and their functional relevance. Plant Biotechnol J 12:253-264. https://doi.org/10.1111/pbi.12133

Kawahara Y, de la Bastide M, Hamilton JP et al (2013) Improvement of the Oryza sativa Nipponbare reference genome using next generation sequence and optical map data. Rice (N Y) 6:4. https://doi.org/10.1186/1939-8433-6-4

Köster J, Rahmann S (2012) Snakemake - a scalable bioinformatics workflow engine. Bioinformatics 28:2520-2522. https://doi.org/10.1093/ bioinformatics/bts480

Lekklar C, Pongpanich M, Suriya-arunroj D et al (2019) Genome-wide association study for salinity tolerance at the flowering stage in a panel of rice accessions from Thailand. BMC Genomics 20:76. https://doi.org/10.1186/ s12864-018-5317-2

Li H, Durbin R (2009) Fast and accurate short read alignment with burrowswheeler transform. Bioinformatics 25:1754-1760. https://doi.org/10.1093/ bioinformatics/btp324

Li H, Handsaker B, Wysoker A et al (2009) The sequence alignment/map format and SAMtools. Bioinformatics 25:2078-2079. https://doi.org/10.1093/ bioinformatics/btp352 
Li W, Zhu Z, Chern M et al (2017) A natural allele of a transcription factor in Rice confers broad-Spectrum blast resistance. Cell 170:114-126.e15. https://doi. org/10.1016/j.cell.2017.06.008

Li Z, Fu BY, Gao YM et al (2014) The 3,000 rice genomes project. Gigascience 3:7. https://doi.org/10.1186/2047-217X-3-7

Mansueto L, Fuentes RR, Borja FN et al (2017) Rice SNP-seek database update: new SNPs, indels, and queries. Nucleic Acids Res 45:D1075-D1081. https:// doi.org/10.1093/nar/gkw1135

Naika M, Shameer K, Mathew OK et al (2013) STIFDB2: an updated version of plant stress-responsive transcription factor database with additional stress signals, stress-responsive transcription factor binding sites and stressresponsive genes in arabidopsis and rice. Plant Cell Physiol 54:1-15. https:// doi.org/10.1093/pcp/pcs185

Ohyanagi H, Ebata T, Huang X et al (2016) OryzaGenome: genome diversity database of wild oryza species. Plant Cell Physiol 57:e1. https://doi.org/10. 1093/pcp/pcv171

Osuna-Cruz CM, Paytuvi-Gallart A, Di Donato A et al (2018) PRGdb 3.0: a comprehensive platform for prediction and analysis of plant disease resistance genes. Nucleic Acids Res 46:D1197-D1201. https://doi.org/10.1093/ nar/gkx1119

Ouyang S, Zhu W, Hamilton J et al (2007) The TIGR Rice genome annotation resource: improvements and new features. Nucleic Acids Res 35:8-11. https://doi.org/10.1093/nar/gkl976

Sakai H, Lee SS, Tanaka T et al (2013) Rice annotation project database (RAP-DB): an integrative and interactive database for rice genomics. Plant Cell Physiol 54. https://doi.org/10.1093/pcp/pcs183

Sanseverino W, Roma G, De Simone M et al (2010) PRGdb: a bioinformatics platform for plant resistance gene analysis. Nucleic Acids Res 38:D814-D821. https://doi.org/10.1093/nar/gkp978

Silva J, Scheffler B, Sanabria Y et al (2012) Identification of candidate genes in rice for resistance to sheath blight disease by whole genome sequencing. Theor Appl Genet 124:63-74. https://doi.org/10.1007/s00122-011-1687-4

Tian T, Liu Y, Yan H et al (2017) AgriGO v2.0: a GO analysis toolkit for the agricultural community, 2017 update. Nucleic Acids Res 45:W122-W129. https://doi.org/10.1093/nar/gkx382

Van der Auwera GA, Carneiro MO, Hartl C et al (2013) From FastQ data to high confidence variant calls: the genome analysis toolkit best practices pipeline. Curr Protoc Bioinforma 43:11.10.1-11.10.33. https://doi.org/10.1002/ 0471250953.bi1110s43

Wang D, Liu J, Li C et al (2016) Genome-wide association mapping of cold tolerance genes at the seedling stage in Rice. Rice (N Y) 9:61. https://doi.org/ 10.1186/s12284-016-0133-2

Yonemaru Jl, Ebana K, Yano M (2014) HapRice, an SNP haplotype database and a web tool for rice. Plant Cell Physiol 55:1-12. https://doi.org/10. 1093/pcp/pct188

Zhang F, Wu Z-C, Wang M-M et al (2017) Genome-wide association analysis identifies resistance loci for bacterial blight in a diverse collection of indica rice germplasm. PLoS One 12:e0174598. https://doi.org/10.1371/ journal.pone.0174598

Zhang Z, Hu S, He H et al (2016) Information commons for Rice (IC4R). Nucleic Acids Res 44:D1172-D1180. https://doi.org/10.1093/nar/gkv1141

Zhao H, Yao W, Ouyang Y et al (2015) RiceVarMap: a comprehensive database of rice genomic variations. Nucleic Acids Res 43:D1018-D1022. https://doi.org/ 10.1093/nar/gku894

\section{Publisher's Note}

Springer Nature remains neutral with regard to jurisdictional claims in published maps and institutional affiliations.

\section{Submit your manuscript to a SpringerOpen ${ }^{\circ}$ journal and benefit from:}

- Convenient online submission

- Rigorous peer review

- Open access: articles freely available online

- High visibility within the field

- Retaining the copyright to your article

Submit your next manuscript at $\boldsymbol{\nabla}$ springeropen.com 